\title{
Development of an Electrochemical Adenosine Diphosphate (ADP) Sensing Principle Employing ADP-dependent Kinase
}

\author{
Wakako Tsugawa, Hinako Futagami and Koji Sode* \\ Department of Industrial Technology and Innovation, Graduate School of Engineering, \\ Tokyo University of Agriculture and Technology, \\ 2-24-16 Nakacho, Koganei, Tokyo 184-8588, Japan
}

(Received February 18, 2015; accepted August 18, 2015)

Key words: ADP-dependent kinase, screen-printed carbon electrode, ADP-dependent hexokinase, electrochemical assay, ATP-dependent kinase

In this study, a novel electrochemical sensing principle was developed for adenosine diphosphate (ADP), which utilizes ADP-dependent kinase as the key enzyme. In this principle, ADP-dependent hexokinase, glucose-6-phosphate dehydrogenase, and diaphorase are combined in the presence of glucose, nicotinamide adenine dinucleotide, and hexaammineruthenium (III) as the electron mediator. The reaction was conducted in a homogeneous mixture on a disposable screen-printed carbon electrode. The measurement was completed within $80 \mathrm{~s}$, and ADP was measured between 2 and $50 \mu \mathrm{M}$ with a detection limit of $1.9 \mu \mathrm{M}(\mathrm{S} / \mathrm{N}=3)$. This simple, rapid method can be generally applicable as an electrochemical assay for the detection of adenosine-triphosphatedependent kinases.

\section{Introduction}

Kinases constitute a group of enzymes that catalyze the transfer of phosphorus from high-energy, phosphate-donating molecules to diverse substrates. In biological systems, phosphorylation by kinases plays various roles, such as signal transduction, proliferation, differentiation, metabolism, and apoptosis. Currently, in molecular and cellular biology, because of their significant and fundamental functions, it is essential to measure kinase activity. For this aforementioned reason, pharmaceutical industries as well as research laboratories have focused their attention on the analysis of kinase activity. For measuring kinases, it is imperative to develop a versatile method that demonstrates advantages of being homogeneous, robust, and sensitive.

${ }^{*}$ Corresponding author: e-mail: sode@cc.tuat.ac.jp 
Conventionally, adenosine diphosphate (ADP) is measured by the luciferase-luciferin luminescence assay, which utilizes the conversion of ADP to adenosine triphosphate (ATP)..$^{(1,2)}$ Moreover, the measurement of ADP by a colorimetric or fluorometric method is also commercialized. ${ }^{(3)}$ In the presence of an "enzyme mixture," ADP is converted to an intermediate, and the intermediate reduces the probe reagent, which is colorless, to a colored product. Kumagai et al. have reported a fluorometric assay for the detection of ADP by utilizing the coupling reaction of ADP-dependent hexokinase (ADP-HK), glucose-6-phosphate dehydrogenase (G6PDH), and diaphorase. ${ }^{(4)}$ In combination with enzymes, resazurin was converted to resorufin, and resorufin-derived fluorescence was measured.

Despite these published studies, colorimetric, fluorometric, and luminescence assays suffer from a limitation in that these assays are prone to interference by colored or turbid samples; hence, electrochemical assays are preferable. Ruan et al. have used an ionicliquid-modified carbon electrode and reported the electrochemical detection of ADP. ${ }^{(5)}$ However, in their study, a high potential of $+1.381 \mathrm{~V}$ was applied, which would oxidize the other electrochemicaly active substances present as contaminants in the sample. Therefore, the combination of enzymes, relatively low potential electron mediators and an electrochemical method will prevent interference from turbidity.

Several ATP-dependent kinases have been utilized for enzymatic analyses of chemicals. Among these kinases, hexokinase (HK) combined with nicotinamide adenine dinucleotide (NAD)-dependent G6PDH for the monitoring of glucose is the most representative and successive method. ${ }^{(6)}$ Because of its high sensitivity and accuracy, this method is recognized as the gold standard for glucose measurement. In this case, it is inevitable to combine $\mathrm{NAD}(\mathrm{P})$-dependent dehydrogenases, which catalyze the oxidation of phosphorylated compounds, for the development of an enzymatic assay, since both products of the HK catalyzing reaction, ADP and glucose-6-phosphate, cannot be directly detected. Thus, an HK-G6PDH system is combined with diaphorase and a color developing dye ${ }^{(7)}$ or an artificial electron acceptor (mediator) for realizing colorimetric or electrochemical sensors for glucose. In other words, colorimetric and/or electrochemical sensors based on the kinase reaction can only be developed when dehydrogenases or oxidases toward phosphorylated compounds as the products of the kinase reaction are available. However, the number and variation of such dehydrogenases or oxidases are limited taking into account the broad applications of several kinases against the molecules to be measured.

On the other hand, some unique enzymes, with which glucose and fructose-6phosphate are phosphorylated using ADP instead of ATP, have been reported. ${ }^{(8-12)}$ These enzymes are known as ADP-dependent kinases. By focusing on the unique reactions that are catalyzed by this group of enzymes, some enzymatic assays have been already developed. ${ }^{(13)}$ However, to the best of our knowledge, enzyme electrodes utilizing ADPdependent kinases have not developed thus far.

In this study, we report a novel electrochemical sensing principle for ADP, which utilizes an ADP-dependent kinase as the key enzyme (Fig. 1). In this principle, ADPdependent hexokinase (ADP-HK), G6PDH, and diaphorase are combined in the presence of glucose, NAD, and hexaammineruthenium(III) (Ru complex) as the electron mediator. 


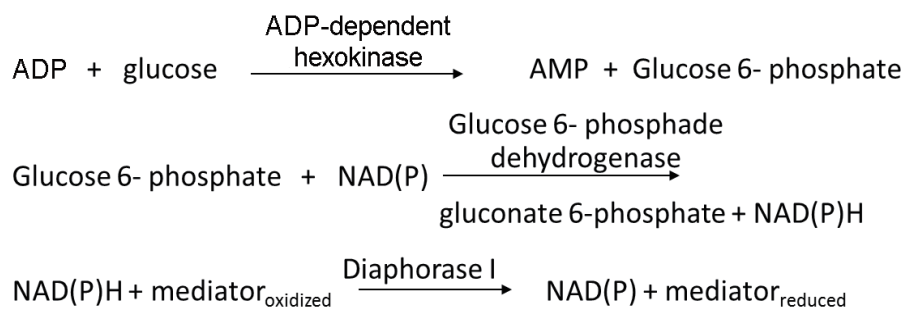

Fig. 1. Schematic of an electrochemical ADP sensing principle utilizing ADP-dependent hexokinase. The ADP-dependent hexokinase reaction liberates glucose-6-phosphate. Glucose6-phosphate is then oxidized to gluconate-6-phosphate by G6PDH coupled with the reduction of NAD to NADH. NADH is then oxidized to NAD coupled with electron-mediated reduction by diaphorase. On the electrode, the reduced electron mediator is then oxidized, and the anodic current correlates with the concentration of the reduced electron mediator. In the presence of a series of enzymes, NAD, electron mediator, and an excess amount of glucose, the concentration of the reduced electron mediator reflects the ADP concentration.

The reaction was conducted in a homogeneous mixture containing an appropriate concentration of each molecule to be detected by a disposable screen-printed carbon electrode. The measurement was completed within $80 \mathrm{~s}$, and ADP was measured between 2 and $50 \mu \mathrm{M}$ with a detectable limit of $1.9 \mu \mathrm{M}$.

\section{Materials and Methods}

\subsection{Chemicals}

ADP-HK from Pyrococcus furiosus was supplied by Asahi Kasei Pharma (Japan). G6PDH and diaphorase I from B. stearothermophilus were supplied by Unitika (Japan). $\mathrm{Ru}$ complex was purchased from Sigma-Aldrich (USA). NAD was purchased from Wako Pure Chemicals (Japan). All other reagents were of analytical grade, and pure water or distilled water was used.

\subsection{Analytical procedure}

Electrochemical measurements were conducted on a Hokuto Denko (Japan) electrochemical analyzer, HZ3000 or HSV-100. In all electrochemical experiments, a screen-printed carbon electrode composed of a three-electrode system, equipped with a working electrode of $2.4 \mathrm{~mm}^{2}$, a carbon-printed auxiliary electrode of $4.8 \mathrm{~mm}^{2}$, and a $\mathrm{Ag} / \mathrm{AgCl}$ ink-printed reference electrode. All measurements were conducted using a disposable new screen-printed carbon electrode at room temperature.

An electrochemical sensor system using a disposable electrode is attractive from the viewpoint of being simple and rapid, as well as being suitable for on-site applications. Hence, we designed this system in which ADP measurement could be completed in 
approximately $1 \mathrm{~min}$. The "reagent solution" is composed of $50 \mathrm{mM}$ glucose, $5 \mathrm{mM}$ NAD, $40 \mathrm{mM}$ Ru complex, $2.5 \mathrm{U} / \mathrm{mL}$ ADP-HK, $25 \mathrm{U} / \mathrm{mL}$ G6PDH, and $125 \mathrm{U} / \mathrm{mL}$ diaphorase. The hexokinase reaction is the initial step of the sequential enzyme reactions; this step must not be the rate-limiting step of these multienzyme reactions. Hence, hexokinase was added in excess, with a glucose concentration of $50 \mathrm{mM}$, sufficient for $V_{\max }$ based on the $K_{\mathrm{m}}$ value of ADP-HK $(0.64 \mathrm{mM})$. $^{(14)}$ The concentration of the Ru complex was decided according to the screen-printed carbon electrode glucose sensor. ${ }^{(15)}$ The ratio between G6PDH and diaphorase was 1:5, as determined by the reference ratio of dehydrogenase to diaphorase utilized in an aldehyde sensor employing NAD-dependent aldehyde dehydrogenase and diaphorase. ${ }^{(16)}$

For a typical reaction, $8 \mu \mathrm{L}$ of the reagent solution, the final concentration of which contains $50 \mathrm{mM}$ glucose, $2.5 \mathrm{U} / \mathrm{mL}$ ADP-HK, 0.1-5 mM NAD, $25 \mathrm{U} / \mathrm{mL}$ G6PDH, 125 $\mathrm{U} / \mathrm{mL}$ diaphorase, $2.0 \mathrm{mM} \mathrm{MgCl}_{2}$, and $40 \mathrm{mM} \mathrm{Ru}$ complex in $100 \mathrm{mM}$ Tris- $\mathrm{HCl}(\mathrm{pH}$ 7.5) buffer, was mixed with $2 \mu \mathrm{L}$ of various concentrations of ADP such that the final concentration was $1-50 \mu \mathrm{M}$ (time 0 ). After vortex mixing, $2 \mu \mathrm{L}$ of the solution was loaded onto the electrode. Next, 1 min after "time 0", a potential of $+400 \mathrm{mV}$ vs Ag/ $\mathrm{AgCl}$ was applied, and the current was recorded at $1 \mathrm{~s}$ intervals. All assays were carried out in triplicate.

\section{Results and Discussion}

Figure 2 shows the representative response curves of the screen-printed carbon electrode with the application of a potential of $400 \mathrm{mV} v \mathrm{Ag} / \mathrm{AgCl}$ toward the sample solution containing various concentrations of ADP, $40 \mathrm{mM}$ glucose, $2.0 \mathrm{U} / \mathrm{mL}$ ADP$\mathrm{HK}, 0.08 \mathrm{mM} \mathrm{NAD}, 20 \mathrm{U} / \mathrm{mL}$ G6PDH, $100 \mathrm{U} / \mathrm{mL}$ diaphorase, $1.6 \mathrm{mM} \mathrm{MgCl}_{2}$, and 32

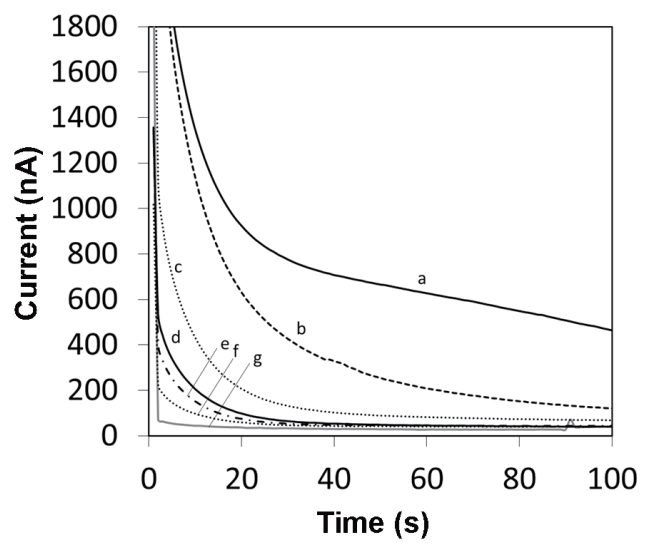

Fig. 2. Representative chronoamperometric responses of the screen-printed carbon electrode toward various ADP concentrations. The sensor response was obtained using a reaction mixture incubated for $1 \mathrm{~min}$ in $0.1 \mathrm{~mol} / \mathrm{L}$ phosphate-buffered saline $(\mathrm{pH}=7.4)$ with $0.08 \mathrm{mM} \mathrm{NAD}$ at +400 $\mathrm{mV}$ (vs Ag/AgCl) containing (a) 100, (b) 50, (c) 10, (d) 5, (e) 2, (f) 1, and (g) $0 \mu \mathrm{mol} / \mathrm{L}$ ADP. 
$\mathrm{mM} \mathrm{Ru}$ complex after $1 \mathrm{~min}$ of incubation at room temperature. The current peak was observed in less than $1 \mathrm{~s}$, after which the current gradually decreased. The current at each time point correlated with the ADP concentration of the measured sample. The current at $20 \mathrm{~s}$ after the potential was applied was utilized for the quantification of ADP.

Considering that the NAD concentration might affect the rate of the reaction of G6PDH and diaphorase, and consequently change the dynamic range and sensitivity of ADP measurement, the electrochemical measurement of ADP was conducted at different NAD concentrations. Figure 3 shows the effect of NAD concentration at ADP concentrations of 1 and $0 \mathrm{mM}$ on the current response of the sensor. With the increase in NAD concentration to a value greater than $4 \mathrm{mM}$, the current toward $1 \mathrm{mM}$ ADP decreased because the equilibrium for the reaction of diaphorase shifted toward the production of NADH. Hence, we selected a NAD concentration of $4.0 \mathrm{mM}$.

Considering that the current at $0 \mathrm{mM}$ ADP was very low, the possibility of electron transfer between the Ru complex and other compounds, such as glucose, G6PDH, gluconate-6-phosphate, the product of the G6PDH reaction, ADP, and AMP, is almost negligible. Hence, the current observed when ADP was added to the reaction mixture is attributed to the sequential reaction of ADP dephosphorylation, glucose phosphorylation, glucose-6-phosphate oxidation, NAD reduction, and the reduction and re-oxidation of the $\mathrm{Ru}$ complex on the electrode, not by the direct oxidation of the $\mathrm{Ru}$ complex or the $\mathrm{Ru}$-complex-mediated oxidation of those components in the reaction mixture.

Figure 4 shows the correlation between the ADP concentration and the sensor current observed $20 \mathrm{~s}$ after the application of a potential of $400 \mathrm{mV}$ vs $\mathrm{Ag} / \mathrm{AgCl}$. Each ADP
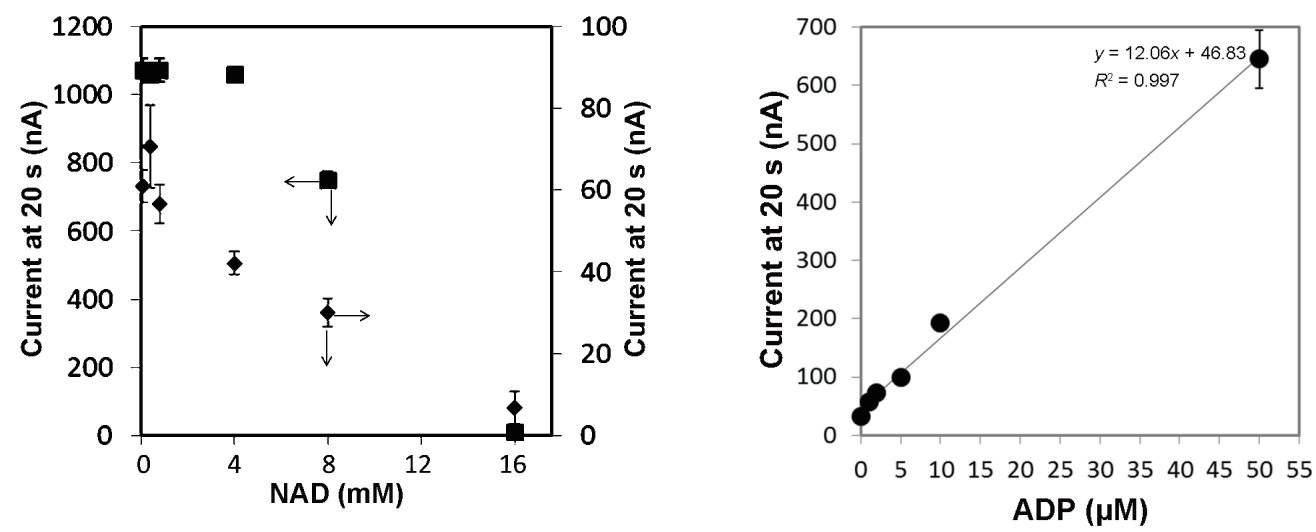

Fig. 3 (left). Effect of NAD concentration on the sensor response. The current for $1 \mathrm{mM}$ ADP (closed squares) or $0 \mathrm{mM}$ ADP (closed diamonds) observed $20 \mathrm{~s}$ after the application of potential is plotted against the final concentration of NAD on the sensor. Error bars represent the standard deviation for each ADP concentration $(n=3)$.

Fig. 4 (right). ADP calibration curve in the presence of $4.0 \mathrm{mM} \mathrm{NAD.} \mathrm{The} \mathrm{current} \mathrm{observed} 20$ $\mathrm{s}$ after the application of potential is plotted against ADP concentration. Error bars represent the standard deviation for each ADP concentration $(n=3)$. 
solution was incubated in the solution, the final concentration of which contains $4.0 \mathrm{mM}$ NAD, $40 \mathrm{mM}$ glucose, $2 \mathrm{U} / \mathrm{mL}$ ADP-HK, $20 \mathrm{U} / \mathrm{mL}$ G6PDH, $100 \mathrm{U} / \mathrm{mL}$ diaphorase, and $32 \mathrm{mM} \mathrm{Ru}$ complex, and incubated for $1 \mathrm{~min}$, and then the potential was applied. A good linear correlation was observed between the ADP concentration from 2 to $50 \mu \mathrm{M}$ and the current at a time of $20 \mathrm{~s}$. The detection limit for ADP was $1.9 \mu \mathrm{M}(\mathrm{S} / \mathrm{N}=3)$. These results indicate that during a coupled enzyme reaction of this sensing principle, the rate limiting step is the ADP-HK reaction. The measurement exhibited good reproducibility for $2 \mu \mathrm{M}$ ADP with a coefficient of variation (CV) of $3.5 \%$ and $5 \mu \mathrm{M}$ ADP with a $\mathrm{CV}$ of $4.9 \%$.

Several ATP-dependent kinases have been reported to be used for the simple and sensitive detection of chemicals such as acetate and creatine, utilizing acetate kinase and creatine kinase, respectively. ${ }^{(17,18)}$ The dynamic range required in these previous studies has indicated that approximately $10-100 \mu \mathrm{M}$ ADP is released taking into account the designed reaction. As our proposed system can be combined with any of the ATP kinases that release ADP, a novel electrochemical sensing system such as for acetate and creatine can be constructed in the future. In addition, a sensor strip with very stable chemical components can be developed, as our system can employ thermostable ADP-HK and diaphorase, both of which are derived from thermophilic microorganisms, and various thermostable G6PDHs can be available.

\section{Conclusions}

In this paper, we report a novel electrochemical principle for the sensing of ADP by utilizing ADP-HK, G6PDH, diaphorase, and a Ru complex as the electron mediator. By this method, ADP can be measured in only $80 \mathrm{~s}$ with a detection limit for ADP as low as 1.9 $\mu \mathrm{M}(\mathrm{S} / \mathrm{N}=3)$. In combination with general ATP-dependent kinases, kinase activity can be measured, and various substances, serving as the substrate of ATP-dependent kinases, can be measured. As the measurement procedure is simple and facile owing to the use of a disposable screen-printed carbon electrode, it demonstrates practical utility for rapid on-site determination.

\section{Acknowledgements}

We thank Asahi Kasei Pharma Corporation and Unitika Limited for providing enzyme samples.

\section{References}

1 S. Murakami, K. Ito, T. Goto, S. Kamada and M. Maeda: Anal. Chim. Acta 361 (1998) 19.

2 M. Helenius, S. Jalkanen and G. G. Yegutkin: Biochim. Biophys. Acta 1823 (2012) 1967.

3 BioAssay Systems: EnzyLight ${ }^{\mathrm{TM}}$ ADP/ATP Ratio Assay Kit, https://www.bioassaysys.com/ view/images/Datasheet/ELDT.pdf (accessed July 2015).

4 K. Kumagai, H. Kojima, T. Okabe and T. Nagano: Anal. Biochem. 447 (2014) 146.

5 C. Ruan, Z. Sun, S. Lu, L. Li, J. Lou and W. Sun: Russ. J. Electrochem. 50 (2014) 129.

6 J. I. Petersona and D. S. Young: Anal. Biochem. 23 (1968) 301.

7 H. J. Coburn and J. J. Carroll: Clin. Chem. 19 (1973) 127. 
8 V. Guixé and F. Merino: IUBMB Life 61 (2009) 753.

9 S. W. Kengen, F. A. de Bok, N. D. van Loo, C. Dijkema, A. J. Stams and W. M. de Vos: J. Biol. Chem. 269 (1994) 17537.

10 S. W. Kengen, J. E. Tuininga, F. A. de Bok, A. J. Stams and W. M. de Vos: J. Biol. Chem. 270 (1995) 30453.

11 S. Koga, I. Yoshioka, H. Sakuraba, M. Takahashi, S, Sakasegawa, S, Shimizu and T. Ohshima: J. Biochem. 128 (2000) 1079.

12 S. W. Kengen, A. J. Stams and W. M. de Vos: FEMS Microbiol. Rev. 18 (1996) 119.

13 K. Kumagai, H. Kojima, T. Okabe and T. Nagano: Anal. Biochem. 447 (2014) 146.

14 Asahi Kasei Pharma Corporation: ADP-dependent hexokinase [ADP-HKPII], https://www. asahi-kasei.co.jp/shindan/pdf/T-92_catalog.pdf (accessed July 2015).

15 H. Yamaoka and K. Sode: J Diabetes Sci. Technol. 1 ( 2007) 28.

17 C. Trivin, F. Lenoir, J. P. Bretaudière and Ch. Sachs: Clin. Chim. Acta 121 (1982) 43.

18 C. Bayer: Clin. Chem. 39 (1983) 1613. 